of PTSD in low- and middle-income countries. Under-recognition and under-treatment of PTSD, as well as problems with the PTSD construct, may well exist in certain countries; however, these issues are logically distinct and cannot explain the specific pattern of results we obtained. For example, under-treatment cannot explain why prevalence rates based on standardised population surveys are relatively high or low.

Their second concern is about the measurement of exposure to trauma. It is true that we could not distinguish between different exposure types, which we continue to see as an important limitation. But in our analyses - and also when the exposure rates by Benjet et $a l^{2}$ are used - higher rates of trauma exposure were associated with higher prevalence in the expected way. It is not the exposure data but the country vulnerability data that generate the paradox. Vermetten et al do not raise concerns about the measurement of vulnerability.

We disagree with their suggestion that 'drawing strong inferences from this single data-point is not a scientifically sound approach'. Table 2 and Figure 2 in our paper clearly show patterns in the data as a whole that are not reliant on one country. Vermetten et al suggest that 'other analytic approaches do allow rigorous conclusions regarding the cross-national epidemiology of PTSD'. However, the example they give does not involve country-level variables, which are the focus of our analysis. It is not clear to us how their example is relevant to our quite different research question.

So far, we found indications that, regardless of exposure, PTSD and other mental health problems are more often observed in less vulnerable, more affluent countries. ${ }^{1,3}$ The analyses we have used are appropriate to the question asked. Rather than ignoring challenging findings, we believe it is scientifically responsible to explore them further. If reliable, they have potentially far-reaching implications from an international mental health perspective.

1 Dückers MLA, Alisic E, Brewin CR. A vulnerability paradox in the crossnational prevalence of post-traumatic stress disorder. Br J Psychiatry 2016; 209: 300-5.

2 Benjet C, Bromet E, Karam EG, Kessler RC, McLaughlin KA, Ruscio AM, et al. The epidemiology of traumatic event exposure worldwide: results from the World Mental Health Survey Consortium. Psychol Med 2016; 46: 327-43.

3 Dückers MLA, Brewin CR. A paradox in individual versus national mental health vulnerability. J Trauma Stress, in press.

Michel L. A. Dückers, PhD, NIVEL- Netherlands Institute for Health Services Research, Utrecht; and Impact - National Knowledge and Advice Centre for Psychosocial Care Concerning Critical Incidents, Arq Psychotrauma Expert Group, Diemen, The Netherlands. Email: m.duckers@nivel.nl; Eva Alisic, PhD, Monash University Accident Research Centre, Melbourne, Australia; Chris R. Brewin, PhD, University Accident Research Centre, Melbourne, Australla; Chris R. Brewin,
Department of Clinical, Educational and Health Psychology, University College London, London, UK

doi: 10.1192/bjp.209.6.527a

\section{Ethnic density - meaning and implications}

The ecological study by Keown et $a l^{1}$ is undoubtedly of value, both methodologically and in relation to the further exposition as to how 'structural and social issues can shape mental health', as Burns and Rugkåsa ${ }^{2}$ (p. 97) note in their related editorial. However, some clarification of the authors' use and operationalisation of the term 'ethnic density' is required to more fully understand the study's implications and limitations.

The study documents 'a positive association between ethnicity and compulsory in-patient treatment' in urban areas (p. 158), but as the denominator of population analysis is relatively large (divided by primary care trusts (PCTs) with an average population of 350000 ), it is unclear whether 'ethnic density' is defined in their study according to the overall prevalence of different ethnic groups within these relatively large unit PCT populations under study, or whether smaller and more relevant unit neighbourhood-level measures of ethic density have been used.

An important earlier study using such neighbourhood-level measures, by Das-Munshi et al, ${ }^{3}$ demonstrated that 'people resident in neighbourhoods of higher own-group density experience "buffering" effects from the social risk factors for psychosis' (p. 282). As psychotic presentations are more likely to result in compulsory admission, Das-Munshi et al's findings would be expected to predict, when controlling for other variables highlighted by Keown et al - in particular, age and deprivation indices - that higher ethnic density, through 'buffering effects', would lead to lower levels of compulsory admission. Although it is possible that the findings of Das-Munshi et al and Keown et al are therefore in contradiction, it seems more likely that the Keown et al study did not measure ethnic density at the more relevant neighbourhood level in which buffering effects are manifest, and therefore that their measure of 'ethnic density' is less meaningful.

Ecological studies, by definition, attempt to attend to these more proximal influences on the immediate living environment. ${ }^{4}$ Although the data-set used by Keown et al no doubt precluded this, the contingent limitations of such data, if this was the case, are therefore important to further acknowledge. Neighbourhoodlevel ethnic density data would also be needed to confirm the significance of Keown et als unexpected finding of a lack of association between ethnicity and compulsion in rural areas, where genuine neighbourhood-level ethnic density might be expected to be low, at least in some areas. Nonetheless, Keown et al's study alerts us to the importance of attending to both social and cultural factors influencing the genesis, precipitation and maintenance of mental illness, including psychosis, which may be variously protective or risk-amplifying, and which interact in complex - sometimes counterintuitive - ways, influencing prognosis, ${ }^{5}$ hospital admission and compulsion.

1 Keown $\mathrm{P}$, McBride O, Twigg L, Crepaz-Keay D, Cyhlarova E, Parsons $\mathrm{H}$, et al. Rates of voluntary and compulsory psychiatric in-patient treatment in England: an ecological study investigating associations with deprivation and demographics. Br J Psychiatry 2016; 209: 157-61.

2 Burns T, Rugkåsa J. Hospitalisation and compulsion: the research agenda. Br J Psychiatry 2016; 209: 97-8.

3 Das-Munshi J, Bécares L, Boydell JE, Dewey ME, Morgan C, Stansfeld SA, et al. Ethnic density as a buffer for psychotic experiences: findings from a national survey (EMPIRIC). Br J Psychiatry 2012; 201: 282-90.

4 Jadhav S, Jain S, Kannuri N, Bayetti C, Barua M. Ecologies of suffering: mental health in India. Econ Polit Wkly 2015; 50: 12-5.

5 Rodger J, Steel Z. Between Trauma and the Sacred: the Cultural Shaping of Remitting-Relapsing Psychosis in Post-Conflict Timor-Leste. Springer, 2016.

James Rodger, Consultant Child \& Adolescent Psychiatrist, South Devon CAMHS. Email: j.rodger@exeter.ac.uk

doi: 10.1192/bjp.209.6.528

Authors' reply: We thank Rodger for his interest in our study and for the opportunity to clarify our measure of ethnic density. The measure used was the percentage of the total adult PCT population from Black and minority ethnic (BME) groups. However, the original work which preceded this ecological analysis ${ }^{1}$ was a multilevel model to estimate the risk of compulsory admission, which involved simultaneous consideration of both individual ethnicity and ethnic density calculated as the proportion of adults reporting White British ethnicity for lower-layer super output areas (LSOAs; average population $\sim 1500$ ), which we loosely regarded as 'neighbourhoods'. In that study, neighbourhood ethnic density was associated with an increased overall risk of compulsory 
admission, even when controlling for individual ethnicity. Furthermore, we found a dose-response relationship: the greater the percentage of the neighbourhood population from BME groups, the greater the overall risk of compulsory admission. It is important to note that we measured the risk of compulsory admission for all adults attending secondary mental healthcare services in England during 2010/11, rather than the risk of compulsory admission for the entire BME population or for individual ethnic groups.

Rodger also makes reference to the work of Das-Munshi et al, ${ }^{2}$ exploring the buffering effect of 'own-group density' across middle layer super output areas (population average 7200 ). Own-group density and overall ethnic density are two different ways of operationalising area-level ethnicity. Indeed, in our multilevel analysis we also tested the association between risk of compulsory admission and neighbourhood ethnic diversity (mixing) using the Theil index. ${ }^{3}$ A very weak negative association was evident, suggesting that more homogeneous neighbourhoods were associated with an increased risk of compulsory admission, although the association was not statistically significant. Furthermore, the Theil index was strongly negatively correlated with our neighbourhood-level index of White British ethnicity $(r=-0.839, P<0.001)$. For these reasons, we did not include the ethnic diversity findings in our original work. ${ }^{1}$ We agree with Rodger that there are a number of limitations in terms of identifying individual-level factors from a paper looking at associations at a population level. Further studies are needed to discern the different effects of ethnic density, ethnic diversity and the buffering effects of own-group density, all of which are slightly different ways of capturing neighbourhood ethnicity. The findings of our multilevel analysis at LSOA level suggest that any buffering effects of ethnic diversity were outweighed by overall ethnic density.

We agree that the lack of association between ethnicity and compulsory admission at the PCT level in rural areas is intriguing. Defining areas such as communities or neighbourhoods is difficult, and it can be problematic to pick up the effects of buffering. The finding may also be due to the negative correlation between ethnic density and deprivation seen in rural settings.

1 Weich S, McBride O, Twigg L, Keown P, Cyhlarova E, Crepaz-Keay D, et al. Variation in compulsory psychiatric inpatient admission in England: a crosssectional, multilevel analysis. Health Serv Deliv Res 2014; doi: 10.3310/ hsdr02490.

2 Das-Munshi J, Bécares L, Boydell JE, Dewey ME, Morgan C, Stansfeld SA, et al. Ethnic density as a buffer for psychotic experiences: findings from a national survey (EMPIRIC). Br J Psychiatry 2012; 201: 282-90.

3 Massey D, Denton N. The dimensions of residential segregation. Social Focus 1988; 67: 281-315.

Patrick Keown, MRCPsych, Academic Psychiatry, Campus for Ageing \& Vitality, Newcastle University, Newcastle upon Tyne. Email: patrick.keown@newcastle.ac.uk: Orla McBride, PhD, School of Psychology, University of Ulster, County Londonderry, Ulster; Liz Twigg, PhD, Department of Geography, University of Portsmouth, Portsmouth; David Crepaz-Keay, DProf, Mental Health Foundation, London; Eva Cyhlarova, DPhil, Department of Physiology, Anatomy and Genetics, University of Oxford, Oxford; Helen Parsons, PhD, Division of Health Sciences, Warwick Medica School, University of Warwick, Coventry; Jan Scott, MD, Academic Psychiatry, Campus for Ageing \& Vitality, Newcastle University, Newcastle upon Tyne; Kamaldeep Bhui, MD, Centre for Psychiatry, Barts and The London School of Medicine \& Dentistry, Queen Mary University of London, London; Scott Weich, MD, Division of Mental Health and Wellbeing, Warwick Medical School, University of Warwick, Coventry, UK

doi: 10.1192/bjp.209.6.528a 